\title{
Hodgkin's Lymphoma and Human Immunodeficiency Virus Infection
}

\author{
Moosa Patel
}

Additional information is available at the end of the chapter

http://dx.doi.org/10.5772/51671

\section{Introduction}

Hodgkin's lymphoma is a lymphoid neoplasm first described by Thomas Hodgkin in 1832 and subsequently by Samuel Wilks in 1865 (1,2). Greenfield (1878), Sternberg (1879) and Reed (1902) are credited with the earliest descriptions of the pathological characteristics of the disease $(3,4,5)$. In has now become clear that the Reed-Sternberg cell is derived from clonal B-cells, more specifically post-germinal center B-cells, giving credence to the malignant nature of the disease, and hence the preferred term of Hodgkin's lymphoma -HL, instead of Hodgkin's disease (6).

Major and striking advances have been made in the biology and management of HL. More than $70 \%$ of patients with HL are curable (especially those presenting with early stage disease). Better insight has been gained with regard to the acute and long term toxicities of chemotherapy and radiotherapy. Furthermore, the advent of new imaging techniques such as PET (positron emission tomography)-scans are allowing therapy to be individualized and tailored in a risk adapted and response adapted fashion (7).

The incidence of HL varies widely throughout the world (approximately 1-3.5/100 000), based on geographical and ethnic factors. The highest rates of HL are seen in the United States, Canada and Europe, with much lower rates occurring in Japan, Korea and China. HL is more common in males compared to females, with a male to female ratio of $1.5: 1$. HL occurs most often in young adults, with a peak frequency in the third decade of life. A bimodal age distribution may be seen, with a second age peak noted in the $6^{\text {th }}$ to $8^{\text {th }}$ decades $(8,9,10,11)$.

The exact aetiology of HL is unknown. An increased risk of HL is seen with Epstein-Barr Virus (EBV) infection, congenital and acquired immunodeficiency states (such as Human Immunodeficiency Virus - HIV infection/AIDS- Acquired Immunodeficiency Syndrome, 
post solid organ and haematopoietic stem cell transplantation) and autoimmune disease (12-18). There is also an increased risk of familial aggregation of HL (19).

HIV infection is known to be associated with an increased risk of HL, based on linkage and cohort studies. The relative risk is now 10-15 fold higher compared with the general population (13-15,17,18,20-22). This review will focus on HL in southern Africa, and describe the differences compared to HL in developed countries, highlighting the emerging increase in HL in the HIV seropositive population/people living with HIV/AIDS (PWHA).

\section{General aspects of HIV lymphoma}

Lymphomas are known to occur with an increased frequency in PWHA $(20,21,23)$. NHL (Non-Hodgkin's Lymphoma) is the commonest malignancy in the post cART (combination antiretroviral therapy) era in PWHA. The incidence of NHL in resource-rich settings has decreased in the post cART era compared to the pre cART era (23-24). However, the contrary is true in resource-poor settings, with a noticeable increase in NHL incidence (2426). This is particularly true of sub-Saharan Africa - the epicenter of the HIV pandemic. The increase in NHL incidence is due mainly to the high prevalence and heavy burden of HIV in sub-Saharan Africa, a region in which two thirds of PWHA reside, and additionally, in many countries in sub-Saharan Africa, there has been a lack of availability of, or delay in initiation of cART. In South Africa, which is home to over 5 million PWHA, the rollout of cART occurred in 2004, 8 years later than in the Western world (introduced in developing countries during the end of 1996 and the beginning of 1997) and until recently, cART was generally only available to individuals with CD4 counts of $<200 /$ ul. Although this practice has changed in the last two years, with access/availability of cART to individuals with a higher CD4 count of $<350 /$ ul, the burden of diseases related to the immunodeficiency state (with notable exceptions such as Kaposi's sarcoma) appear to be on the increase $(17,25,26,27)$.

In Africa, the HIV/AIDS epidemic was first reported in 1984 (28). The major risk of HIV in Africa occurs in/with heterosexual relationships, and accounts for an approximately equal male to female ratio, as compared to the Western world in which the major risk groups involve intravenous drug use and homosexual relationships, thus predominantly affecting males. Furthermore, early in the HIV epidemic, there was no marked increase in the incidence of NHL compared to the USA. This was attributed to PWHA dying earlier in the course of their disease possibly from infectious complications such as pneumonia and tuberculosis. The decreased longevity prevented the subsequent or later development of NHL. In addition, there may be underreporting of lymphoma or the missed diagnosis of lymphoma, with a diagnosis of an infective cause of lymphadenopathy (such as tuberculosis) being favoured over lymphoma, in the absence of performing a fine needle aspirate or lymph node biopsy (28-30).

At Chris Hani Baragwanath Academic Hospital (CHBAH) - a tertiary, public sector, University of the Witwatersrand linked hospital located in Soweto, Johannesburg, studies in 
the 1990's with respect to HIV-NHL showed only a modest increase of NHL in seropositive individuals, with an odds ratio of 4.8, 5 and 5.9 respectively (31-33). The first patient at this hospital with HIV-NHL was seen in 1993. Since then, there has been a steady increase in HIV-NHL up to 2000. However, since 2001, the percentage seropositivity of NHL has exceeded 50\% (approximately between $60-80 \%$ ), and since 2002, there has additionally been a significant increase in the total number of patients diagnosed with NHL at CHBAH (from 20-30 new patients per year to 70-80 patients per year) (see Table 1 and Figure 1) (25). Indeed, NHL is now the commonest haematological malignancy in South Africa, in the current HIV/AIDS era and the number of seropositive patients continues to increase.

With respect to Hodgkin's lymphoma (HL), the data is less dramatic, but is becoming more significant. In a study by Stein et al, 2008 (33), the percentage seropositivity of HL in a South African cohort (which included patients from $\mathrm{CHBAH}$ ) was $19.5 \%$ (OR=1.6, 95\% CI=1.0-2.7), during the period 1995-2004. The first patient with HIV-HL was seen in 1994 at CHBAH. Since then, there has been a modest increase in HIV-HL up to 2006. However, in the last 5 years (2007 - 2011) at our single institution, the percentage seropositivity is greater than $50 \%$ (see Figure 2) and the number of patients over the years are gradually increasing (doubled compared to an earlier series in the late 1980's and early 1990's - (17,34)(see Figure 3). Thus, the focus of this review relates to the emerging problem of Hodgkin's lymphoma in the setting of HIV in southern Africa.

\begin{tabular}{|c|c|c|c|c|}
\hline Year & $\begin{array}{c}\text { Total Number } \\
\text { of Patients }\end{array}$ & $\begin{array}{c}\text { Number of } \\
\text { Seronegative } \\
\text { Patients }\end{array}$ & $\begin{array}{c}\text { Number of } \\
\text { Seropositive } \\
\text { Patients }\end{array}$ & \% Seropositive \\
\hline 1993 & 20 & 19 & 1 & $5 \%$ \\
\hline 1994 & 20 & 18 & 2 & $10 \%$ \\
\hline 1995 & 18 & 15 & 3 & $16.7 \%$ \\
\hline 1996 & 28 & 25 & 3 & $10.7 \%$ \\
\hline 1997 & 18 & 14 & 4 & $22.2 \%$ \\
\hline 1998 & 34 & 18 & 16 & $47.1 \%$ \\
\hline 1999 & 22 & 14 & 8 & $36.4 \%$ \\
\hline 2000 & 30 & 16 & 14 & $46.7 \%$ \\
\hline 2001 & 24 & 4 & 20 & $83.3 \%$ \\
\hline 2002 & 40 & 15 & 25 & $62.5 \%$ \\
\hline 2003 & 44 & 17 & 27 & $61.4 \%$ \\
\hline 2004 & 58 & 23 & 35 & $60.3 \%$ \\
\hline 2005 & 54 & 15 & 39 & $72.2 \%$ \\
\hline 2006 & 72 & 17 & 55 & $76.4 \%$ \\
\hline 2007 & 72 & 19 & 53 & $73.6 \%$ \\
\hline 2008 & 76 & 10 & 66 & $86.8 \%$ \\
\hline
\end{tabular}

Table 1. Patients with non-Hodgkin's Lymphoma seen at Chris Hani Baragwanath Academic Hospital from 1993 to 2008 


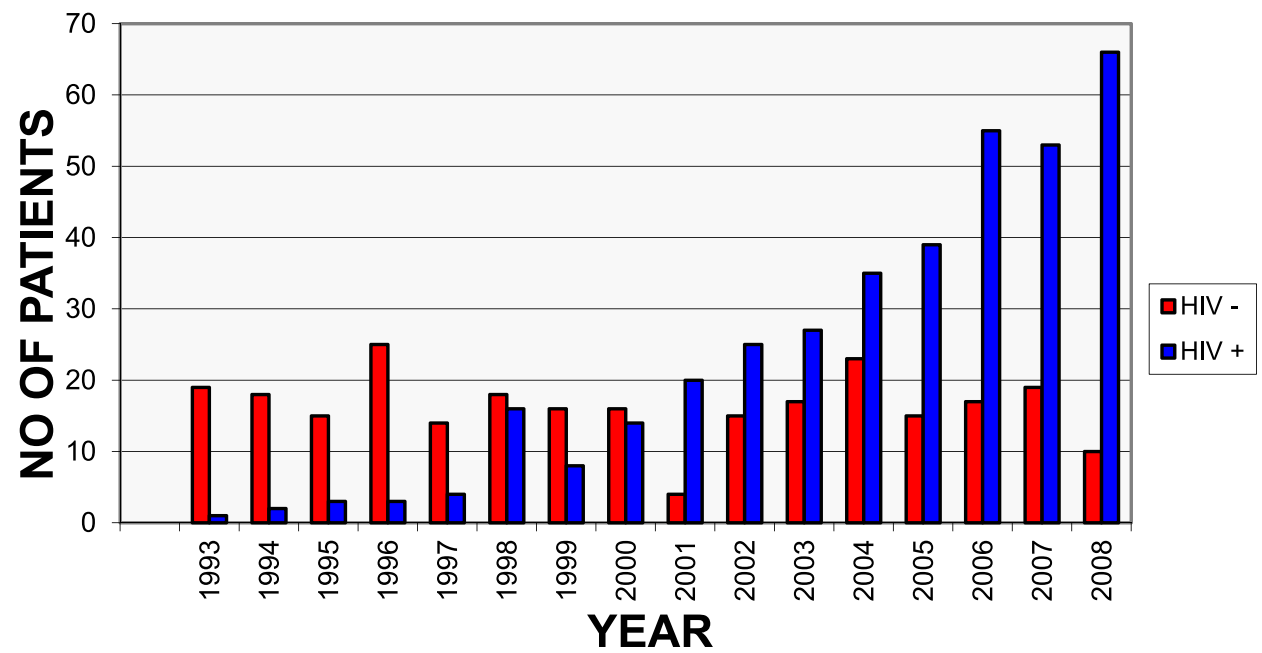

Figure 1. HIV seropositive and HIV seronegative patients with non-Hodgkin's Lymphoma from 1993 to 2008 seen at Chris Hani Baragwanath Academic Hospital

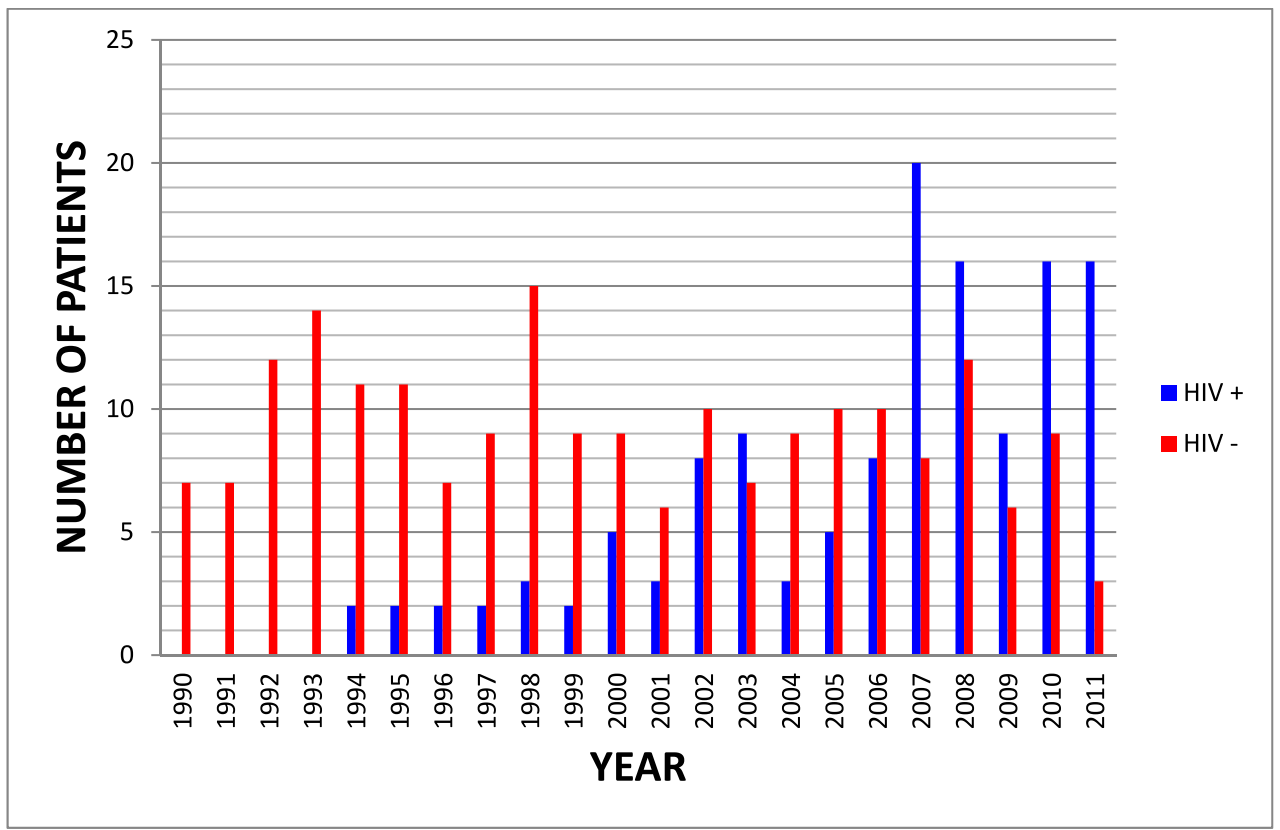

Figure 2. HIV seropositive and HIV seronegative patients with Hodgkin's Lymphoma from 1990 to 2011 seen at Chris Hani Baragwanath Academic Hospital 


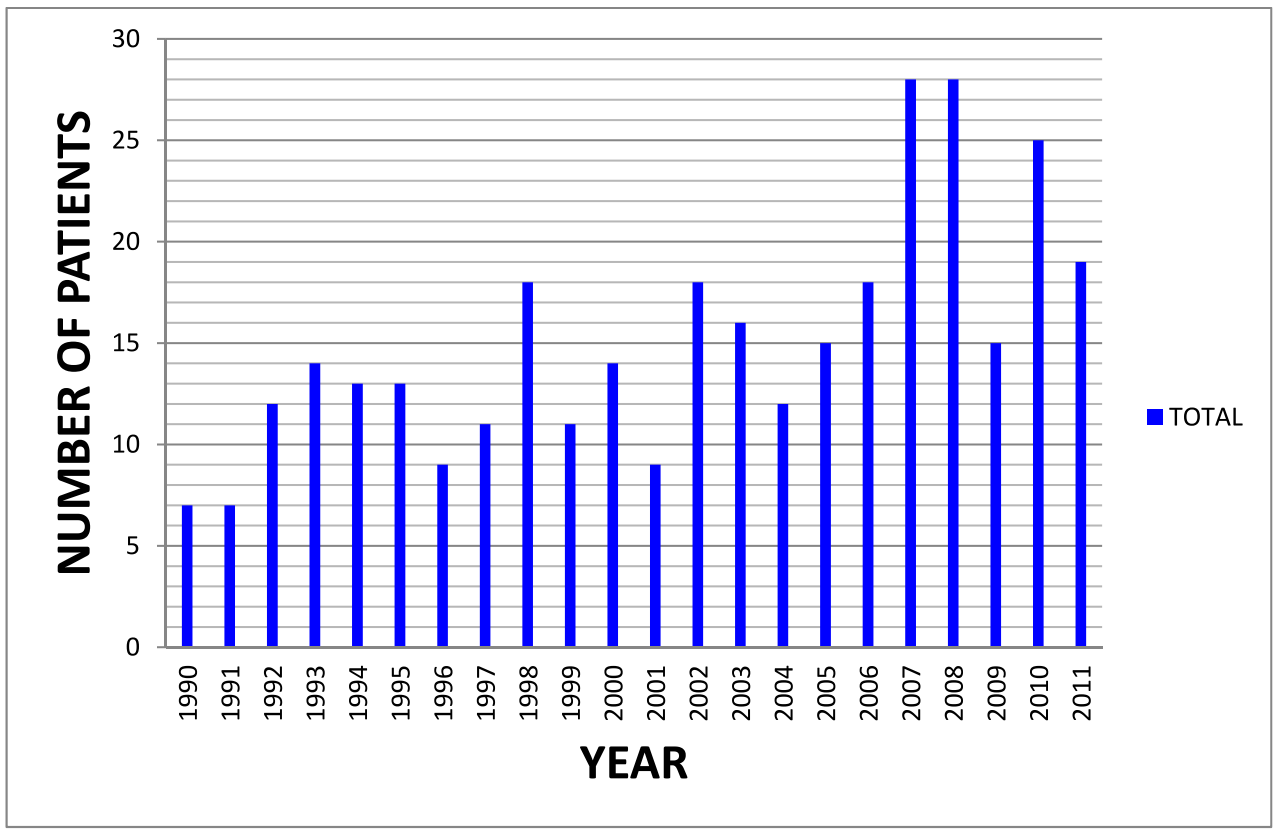

Figure 3. Total number of patients with Hodgkin's Lymphoma seen at Chris Hani Baragwanath Academic Hospital from 1990 - 2011

\section{Hodgkin's lymphoma and HIV}

\subsection{Introduction and epidemiology}

HL occurring in the setting of immunodeficiency (with particular reference to HIV/AIDS) is generally aggressive, presents with advanced stage disease, frequent constitutional (' $\mathrm{B}$ ') symptoms, less favourable histology, more frequent bone marrow involvement and a poorer prognosis compared to immunocompetent individuals $(17,18)$.

With the advent of HAART (highly active antiretroviral therapy), now referred to as cART (combination antiretroviral therapy), the AIDS related morbidity, particularly with respect to opportunistic infections has decreased and the survival of HIV/AIDS patients has increased $(35,36)$. In the post cART era, ADCs (AIDS-defining cancers) continue to fall, but the rates of NADCs (non-AIDS defining cancers) such as HL, anal carcinoma, lung carcinoma and skin cancers are on the increase (37). With HL, there is a noticeably increasing relative risk of approximately $10-15$ fold, compared with the general population $(13-15,17,18,20-22)$.

Several epidemiological studies conducted in the last two decades, and summarised in the article by Carbone et al, 2009 (22) strongly support the evidence that HIV positive individuals have a higher risk of developing HL compared to their HIV negative counterparts. This is in contrast to HIV-NHL or HIV-Kaposi's sarcoma (where the incidence 
of the disease has decreased significantly after the introduction of cART). cART has allowed the use of standard therapeutic options to be delivered to seropositive patients in a more optimal manner, bringing about renewed optimism in the management of such patients. cART use is associated with higher CD4 T cell counts and enhanced immunity. Thus, despite the benefit of cART, which improves immunity and decreases the risk of opportunistic infections, there is a paradoxical increased risk of HL (22). The authors conclude that the improved CD4 T cell count that occurs post cART use, provides antiapoptotic pathways and mechanisms for immune escape by tumour cells, thus resulting in an increased risk of HL (22).

However, in contrast to this, a 20-year cohort study has shown that with the advent of antiretroviral therapy, ADCs (AIDS-defining cancers) continue to fall, but the rates of NADCs (non-AIDS defining cancers) are on the increase. The authors suggest that this increase appears to be more related to the aging of the HIV population (i.e. increased longevity allowing a greater risk of developing lymphoma) rather than the antiretroviral therapy and its effect on the CD4 T cell count (37).

\subsection{Pathogenesis}

Histologically, HL is characterized by a population of neoplastic Reed-Sternberg (RS) cells, which constitute $<1-2 \%$ of the cellular component, admixed with a reactive, mixed inflammatory infiltrate of lymphocytes, plasma cells, eosinophils and histiocytes. Cytokines and chemokines are produced by either the RS cells or the reactive cells in the background micro-environment of the tissue. The cytokine production may explain the presence and maintenance of an impaired immune response, while the chemokines (cytokines with chemoattractant properties) play a role in leucocyte trafficking, attract chemokine receptor CCR4-expressing Th-2 cells and T regulatory cells, and allow a favourable environment for survival of RS cells (38-40). Cross talk between the RS cells and reactive cells mediated by cytokines such as IL-13, IL-17, IL-10, transforming growth factor-beta and chemokines principally CCL17 (thymus and activation-regulated chemokine, TARC) and CCL22 (macrophage-derived chemokine, MDC), lead to an environment where RS cells are able to proliferate, escape from apoptosis and survive host anti-tumour defense (38-40). The CD4+ T cells surrounding the neoplastic cells in HL are CD45RO+/CD45RA-/CD45RB ${ }^{\mathrm{dim}}$, suggesting a memory Th2 phenotype (41).

HIV-associated immunosuppression is a state that permits the unchecked and uncontrolled proliferation of Epstein-Barr virus (EBV) infection. EBV has been implicated in the etiopathogenesis of classic HL, with a high frequency of EBV association (80-100\%) noted in tissues of patients with HIV-HL $(42,43)$. EBV transforming proteins, such as latent membrane protein-1 (LMP-1), is expressed in virtually all HIV-HL patients (43-45). The expression of EBV-LMP-1 is important in the pathogenesis of HIV-HL. LMP-1 expression by EBV-infected RS cells represents the principal mechanism for constitutive NF (nuclear factor) $-\kappa B$ activity, which confers an apoptosis resistant phenotype to the RS cells $(43,45)$. EBV-immortalized B cells also produce CCL17 and CCL22 through LMP-1 mediated activation of NFKB (46). 
RS cells of classical HL represent transformed B cells (post germinal center B cells) that originate from preapoptotic germinal center B cells. They express CD15 and CD30 as well as LMP-1 and display a BCL6-/CD138+/MUM1/IRF4+ (Interferon Regulatory Factor-4) phenotype $(43,47,48)$. In addition, LMP2A and EBNA-1 may also contribute to the development of the RS cells and are expressed in the RS cells of this tumour $(44,45)$. LMP2A may promote the survival of the 'crippled' germinal center B cells, thereby aiding in their development (49).

\subsection{Clinical presentation and management}

In general, HIV-seropositive patients with HL tend to have a more aggressive clinical course than their seronegative counterparts. The behaviour of the disease is different, and based on a number of studies (22,50-57), the following characteristics were noted: more frequent constitutional ' $\mathrm{B}$ ' symptoms - 70-96\%, more advanced stage disease (III and IV) $74-92 \%$, more frequent involvement of extranodal sites $-17-62 \%$, with bone marrow involvement being the most common extranodal site $-40-59 \%$, followed by involvement of the liver $-17-40 \%$ and spleen $-20-30 \%$. The vast majority $(>80 \%)$ of the patients were males. The median age at presentation was approximately 34 years. The median CD4 count was mostly in the intermediate range of $240-306 / \mu \mathrm{l}$ (22,50-57). Compared to HIV negative HL, where nodular sclerosis is the dominant histological subtype, mixed cellularity is most commonly encountered in HIV-HL - 33-53\% (17,22,34,56-57). Nodular sclerosis is the second most common histological subtype in HIV-HL - 24-31\%. However, with more severe immunosuppression, nodular sclerosis becomes infrequent (38). There is also an increasing number of patients with lymphocyte depleted histology - 14-20\% in HIV-HL (17,22,56-57).

Based on the Italian Cooperative Group on AIDS and Tumors (GICAT) study, in comparison with patients who were cART naive, patients receiving cART before the onset of HL are older, have less B symptoms, have higher leukocyte and neutrophil counts and have a higher haemoglobin level (56).

In a recent review of 43 patients with HIV associated HL seen at CHBAH over a 2 year period (July 2008 - June 2010) a number of striking similarities and differences were noted when comparing this cohort with other published studies outside of Africa (17). The median age at presentation of 38 years was similar to other series. There was no striking male predominance. Conversely, the male to female ratio is almost equal at 1.1:1. All the patients had heterosexual acquisition of HIV. None of the patients acquired their HIV through intravenous drug use or homosexual contacts. This is different to other series where homosexuality and intravenous drug use are significant, documented risk groups $(15,20,22,50,56,57)$. The presentation with advanced stage disease $(82 \%)$, more frequent ' $\mathrm{B}$ ' symptoms (93\%), more frequent involvement of extranodal sites (bone marrow-38\%; liver$45 \%$; spleen-28\%) and 'true' extranodal sites (17\%) and the histological pattern of disease (mixed cellularity being the most common) is similar to that reported in the literature. The median CD4 count of $176 / \mu 1$ is generally lower, although there are series reported of HIVHL with median CD4 counts of $<200 / \mu$ l (51). In this series $12 / 29(41 \%)$ of the patients had 
newly diagnosed HIV at the time of the diagnosis of HL. In $62 \%$ of the patients, the duration of the diagnosis of HIV (including new patients was $<1$ year). Only $45 \%$ of the patients were on antiretroviral therapy at diagnosis of HL, compared to $71-80 \%$ in other series $(22,56,57)$. A further striking difference is the high proportion of patients with Tuberculosis in this series $-59 \%$ (38\% with active disease and 21\% with past, documented disease). The high prevalence of tuberculosis may be a reflection of the more severe immunosuppression in the patients, the delay in diagnosis of HIV and hence the absence of antiretroviral therapy use at diagnosis and the very common occurrence of tuberculosis in the general population. The presence of tuberculosis, often in a disseminated fashion, has an adverse impact on the clinical outcome of the patients. In general, the outcome of the HIV-HL patients was less favourable than the HIV seronegative patients (17).

In another local study from CHBAH, covering a fifteen period from 1990 to 2004, the clinical characteristics of 163 patients with Hodgkin's lymphoma are reviewed (58). Table 2 depicts the differences between the HIV seropositive and HIV seronegative patients in this study.

\begin{tabular}{|l|c|c|}
\hline HIV Status & Seropositive & Seronegative \\
\hline Total Number of Patients = 163 & $47(29 \%)$ & $116(71 \%)$ \\
\hline Median Age (Range) in Years & $30(13-59)$ & $29(13-87)$ \\
\hline M:F Ratio & $1.8: 1$ & $1.2: 1$ \\
\hline CD4 Count at Presentation (/ul) & $186(32-769)$ & N/A \\
\hline 'B' Symptoms & $77 \%$ & $78 \%$ \\
\hline Advanced Stage (III \& IV) Disease & $78 \%$ & $67 \%$ \\
\hline 'True' Extranodal Disease & $13 \%$ & $12 \%$ \\
\hline Histological Subtype: & & $42 \%$ \\
Mixed Cellularity & $61 \%$ & $41 \%$ \\
Nodular Sclerosis & $17 \%$ & $13 \%$ \\
Other & $22 \%$ & \\
\hline Tuberculosis & & $23 \%$ \\
Prevalence & $27 \%$ & $17 \%$ \\
Active Disease & $19 \%$ & \\
\hline Treatment Response & $38 \%$ & $57 \%$ \\
Complete Response & $8 \%$ & $21 \%$ \\
Partial Response & & \\
\hline
\end{tabular}

Table 2. Clinical characteristics of HIV seropositive and HIV seronegative patients seen over a fifteen year period (1990 - 2004) at Chris Hani Baragwanath Academic Hospital (adapted from Fazel, 2012)

Based on the findings of this study, HIV seronegative patients compared to HIV seropositive patients have more advanced stage disease, a higher frequency of mixed cellularity subtype, a slightly higher risk of tuberculosis and 'true' extranodal disease and a poorer response to treatment (58).

The management of HIV-HL is challenging because of the frequency of infections, likelihood of organ dysfunction due to HIV, more frequent involvement of the bone marrow, increased 
myelosuppression, potential drug-drug interactions of the antiretrovirals and anti-infectives with chemotherapy, the advanced and widespread nature of the disease at presentation and the preponderance of less favourable histological subtypes. Treatment approaches include vigorous supportive care (HAART, antivirals, antifungals, neutrophil-stimulating growth factors), together with standard multiagent chemotherapy.

Chemotherapy regimens for HIV-HL such as EBV, EBVP, ABVD and MOPP/ABV hybrid are feasible and can be delivered with concomitant cART. The AIDS Clinical Trials Group (ACTG) treated 21 patients with ABVD for 4-6 cycles with G-CSF support. Antiretroviral therapy was not used. The complete remission rate (CR) was $43 \%$ with a median overall survival of 18 months (59). In a more recent Spanish study (GESIDA - Groupo de Estudia de SIDA), 62 patients with HIV-HL received the standard, full-dose ABVD and cART with $87 \%$ of the patients achieving a CR. The 5-year overall survival (OS) and event-free survival (EFS) probabilities were 76 and $71 \%$ respectively. The immunological response to HAART had a positive impact on OS $(\mathrm{p}=0.002)$ and EFS ( $\mathrm{p}=0.001)(60)$. Use of cART substantially improves the overall survival in HIV associated HL. This is due to a decrease in the incidence of opportunistic infections, the ability to deliver more appropriate and aggressive chemotherapy on schedule and to the less aggressive presentation of lymphoma in patients on cART, in comparison with those lymphomas that arise in patients who never received cART (50-53). In the study of Hentrich et al, 2006, 34/59 patients receiving cART ( $n=34)$ had a significantly better 2-year overall survival than those not receiving cART (74\% versus 30\%, $\mathrm{p}<0.001)(61)$. The advent of cART also allows for more aggressive treatment options such as VEBEP (62), BEACOPP (63), Stanford V (64) and the use of high-dose chemotherapy and autologous stem cell transplantation (ASCT) in selected patients $(65,66)$. However, in general, response rates and cure rates are lower than in HIV seronegative patients, despite the substantial progress made in the last decade. The challenge at present is to optimise the use of standard approaches as used in HIV negative HL. Once this is established, evaluation of experimental and newer therapies should follow.

\section{Conclusion}

HIV is associated with an increased risk of developing HL, a risk that has not lessened despite the introduction and benefit of cART. HL is now being regarded among the most common NADCs, which have clearly increased in the post cART era. The association from being largely coincidental (overlapping and similar age group for both HL and HIV) may now be increasingly causal, with the most plausible explanation being attributed to the pathogenetic role of Epstein Barr virus infection.

The recognition of an increasing trend of HIV-HL in resource-poor settings needs to be further highlighted, so that early diagnosis, early recourse to cART and appropriate supportive therapy and specific therapy such as chemotherapy can be administered to improve survival.

Therapy of HIV associated HL entails using the same therapeutic approaches as in seronegative $\mathrm{HL}$, including standard chemotherapy regimens such as ABVD, and in the 
salvage setting, autologous stem cell transplantation in selected patients. In general, the prognosis and overall survival still remains poorer in HIV-HL compared to HIV negative HL. Importantly, the concomitant use of anti-retroviral agents has allowed for the delivery of full-dose and dose-intensive chemotherapy given on schedule, as well as prophylaxis against certain opportunistic infections such as Pneumocystis jirovecii pneumonia, and the liberal use of growth factors (granulocyte colony stimulating factor) and other supportive measures, constitutes an important aspect of supportive therapy and has contributed to an improvement in prognosis. The early recognition and treatment of tuberculosis cannot be overemphasized in settings where tuberculosis is endemic. Newer specific treatment approaches for HL may become necessary in the future to improve survival. However, for the present, HIV-associated HL appears to be on the increase and remains an emerging and ongoing challenge.

\section{Author details}

Moosa Patel

Clinical Haematology Unit, Department of Medicine, Chris Hani Baragwanath Academic Hospital and Faculty of Health Sciences, University of the Witwatersrand, Johannesburg, South Africa

\section{Acknowledgement}

I thank all the medical, nursing and allied healthcare professionals who were involved in the diagnosis, management and follow up of the patients with Hodgkin's lymphoma. In particular, I extend my sincere gratitude to the staff of the Clinical Haematology Unit, Department of Medicine, CHBAH, and all the patients whose data has been used in this study.

\section{References}

[1] Hodgkin T. On some morbid appearances of the absorbent glands and spleen. MedicoChirug Trans 17:68, 1832.

[2] Wilks S. Cases of the enlargement of the liver and spleen (or Hodgkin's disease) with remarks. Guys Hosp Rep 11:56, 1865.

[3] Greenfield WS. Specimens illustrative of the pathology of lymphadenoma and leucocythemia. Trans Pathol Soc Lond 29:272, 1878.

[4] Sternberg C. Uber eine eigenartige unter dem Bilde der Pseudoleukamie verlaufende Tuberculose des lymphatischen Apparates. Ztschr Heilk 19:21, 1879.

[5] Reed D. On pathological changes in Hodgkin's disease, with special reference to its relation to tuberculosis. Johns Hopkins Hosp Rep 10:133, 1902.

[6] Marafioti T, Hummel M, Foss H-D, et al. Hodgkin and Reed-Sternberg cells represent an expansion of a single clone originating from a germinal center B-cell with functional immunoglobulin gene rearrangement but defective immunoglobulin transcription. Blood 95:1443-50, 2000. 
[7] Evens AM and Horning SJ. Hodgkin lymphoma: epidemiology, diagnosis, and treatment. In Management of Hematologic Malignancies. Ed. S O'Brien, JM Vose and HM Kantarjian. Cambridge University Press pp 367-403, 2011.

[8] Katanoda K and Yako-Suketomo H. Comparison of time trends in Hodgkin and NonHodgkin lymphoma incidence (1973-97) in East Asia, Europe and USA, from cancer incidence in five continents, Vol. IV-VIII. Jpn J Clin Oncol 38:391-3, 2008.

[9] http://www.seer.cancer.gov/csr/1975_2005/results_single/sect_01_table.01.pdf.

[10] Correa P and O'Connor GT. Epidemiologic patterns of Hodgkin's disease. Int J Cancer 8:192-201, 1971.

[11] Macfarlane GJ, Evstifeeva T, Boyle P, et al. International patterns in the occurrence of Hodgkin's disease in children and young adult males. Int J Cancer 61:165-9, 1995.

[12] Brousset P, Knecht H, Rubin B, et al. Demonstration of Epstein-Barr virus replication in Reed-Sternberg cells of Hodgkin's disease. Blood 82:872-6, 1993.

[13] Herida M, Mary-Krause M, Kaphan R, et al. Incidence of non-AIDS-defining cancers before and during the highly active antiretroviral therapy era in a cohort of human immunodeficiency virus-infected patients. J Clin Oncol 21:3447-53, 2003.

[14] Biggar RJ, Jaffe ES, Goedert JJ, et al. Hodgkin lymphoma and immunodeficiency in persons with HIV/AIDS. Blood 108:3786-91, 2006.

[15] Hessol NA, Katz MH, Liu JY, et al. Increased incidence of Hodgkin disease in homosexual men with HIV infection. Annals of Internal Medicine 117(4):309-11, 1992.

[16] Landgren O, Engels EA, Pfeiffer RM, et al. Autoimmunity and susceptibility to Hodgkin lymphoma: a population-based case-control study in Scandinavia. J Natl Cancer Inst 98;1321-30, 2006.

[17] Patel M, Philip V and Fazel F. Human immunodeficiciency virus infection and Hodgkin's Lymphoma in South Africa - An emerging problem. Advances in Hematology Volume 2011 (2011), doi:10.1155/2011/578163, 2011.

[18] Spina M, Carbone A, Gloghini A, et al. Hodgkin's Disease in Patients with HIV infection. Advances in Hematology Volume 2011 (2011), doi:10.1155/2011/402682, 2011.

[19] Hjalgrim H, Rasmussen S, Rostgaard K, et al. Familial clustering of Hodgkin lymphoma and multiple sclerosis. J Natl Cancer Inst 96:780-4, 2004.

[20] Lyter D, Bryant J, Thackeray R, et al. Incidence of human immunodeficiency virus related and non related malignancies in a large cohort of homosexual men. J Clin Oncol 13:2540-6, 1995.

[21] Goedert J, Cote T, Virgo P, et al. Spectrum of AIDS-associated malignant disorders. Lancet 351:1833-9, 1998.

[22] Carbone A, Gloghini A, Serraino D, et al. HIV-associated Hodgkin lymphoma. Current Opinion in HIV and AIDS 4:3-10, 2009.

[23] Ziegler JL, Drew WL and Miner RC. Outbreak of Burkitt's-like lymphoma in homosexual men. The Lancet 2(8299):631-33, 1982.

[24] Ulrickson M, Press OW and Casper C. Epidemiology, Diagnosis, and Treatment of HIVAssociated Non-Hodgkin's Lymphoma in Resource Limited Settings. Advances in Hematology Volume 2012(2012), doi:10.1155/2012/932658, 2012. 
[25] Patel M, Philip V, Turton D, et al. The impact of HIV on Non-Hodgkin's Lymphoma at Chris Hani Baragwanath Hospital. Haematologica 92(s1):273, 2007.

[26] UNAIDS. Report on the global AIDS epidemic. Geneva: UNAIDS, 2008.

[27] Casper C. The increasing burden of HIV-associated malignancies in resource-limited regions. Annual Review of Medicine 62:157-170, 2011.

[28] Clumeck N, Sonnet J and Taelman H. Acquired immunodeficiency syndrome in African patients. NEJM 310(8):492-97, 1984.

[29] Parkin DM, Wabinga S, Nambooze S, et al. AIDS-related cancers in Africa: maturation of the epidemic in Uganda. AIDS 13(18):2563-70, 1999.

[30] Adeniji KA and Anjorin AS. Peripheral lymphadenopathy in Nigeria. African Journal of Medicine and Medical Sciences 29(3-4):233-37, 2000.

[31] Sitas F, Bezwoda WR, Levin V, et al. Association between human immunodeficiency type 1 infection and cancer in the black population of Johannesburg and Soweto, South Africa. Br J Cancer 75:1704-07, 1997.

[32] Sitas F, Pacella-Norman R, Carrara H, et al. The spectrum of HIV-1 related cancers in South Africa. Int J Cancer 88,489-92, 2000.

[33] Stein L, Urban MI, O'Connell D, et al. The spectrum of human immunodeficiency virus associated cancers in a South African black population: Results from a case-control study, 1995-2004. Int J Cancer 122:2260-2265, 2008.

[34] Patel M. Haematology. In: Baragwanath Hospital 50 years - A Medical Miscellany. Eds. K Huddle and A Dubb. Ultra Litho. 173-190, 1994.

[35] Ribera JM, Navarro JT, Oriol A, et al. Prognostic impact of highly active antiretroviral therapy in HIV-related Hodgkin's disease. AIDS 16(14):1973-6, 2002.

[36] Gerard L, Galicier L, Boulanger E, et al. Improved survival in HIV-related Hodgkin's lymphoma since the introduction of highly active antiretroviral therapy. AIDS 17(1):8187, 2003.

[37] Crum-Cianflone N, Hullsiek KH, Marconi V et al. Trends in the incidence of cancers among HIV-infected persons and the impact of antiretroviral therapy: a 20-year cohort study. AIDS 23:41-50, 2009.

[38] Takegawa S, Jin S, Nakayama T et al. Expression of CCL17 and CCL22 by latent membrane protein 1-positive tumor cells age-related Epstein-Barr virus-associated B lymphoproliferative disorder. Cancer Sci 99:296-302, 2008.

[39] Niens M, Visser L, Nolte IM et al. Serum chemokine levels in Hodgkin lymphoma patients: highly increased levels of CCL17 and CCL22. British Journal of Haematology 140:527-536, 2008.

[40] Maggio E, van den Berg A, Diepstra A et al. Chemokines, cytokines and their receptors in Hodgkin's lymphoma cell lines and tissues. Annals of Oncology 13(Suppl. 1):52-56, 2002.

[41] Poppema S, Lai R, Visser L et al. CD45 (leucocyte common antigen) expression in T and B lymphocyte subsets. Leuk Lymphoma 20:217-222, 1996.

[42] Rezk SA and Weiss LM. Epstein-Barr virus-associated lymphoproliferative disorders. Human Pathology 38(9):1293-1304, 2007. 
[43] Carbone A, Gloghini A, Larocca LM, et al. Human immunodeficiency virus-associated Hodgkin's disease derives from postgerminal center B cells. Blood 93:2319-2326, 1999.

[44] Young LS and Rickinson AB. Epstein-Barr virus: 40 years on. Nat Rev Cancer 4:757-768, 2004.

[45] Carbone A, Gloghini A, Dotti G. EBV-associated lymphoproliferative disorders: classification and treatment. Oncologist 13:577-585, 2008.

[46] Nakayama T, Hieshima K, Nagakubo D et al. Selective induction of Th2-attracting chemokines CCL17 and CCL22 in human B cells by latent membrane protein1 of Epstein-Barr Virus. J Virol 78:1665-74, 2004.

[47] Klein U and Dalla-Favera R. Germinal centres: role in B-cell physiology and malignancy. Nat Rev Immunol 8:22-33, 2008.

[48] Carbone A, Gloghini A, Larocca LM, et al. Expression profile of MUM1/IRF4, BCL-6, and CD138/syndecan-1 defines novel histogenic subsets of human immunodeficiency virus-related lymphomas. Blood 97:744-751, 2001.

[49] Mancao C and Hammerschmidt W. Epstein-Barr virus latent membrane protein 2A is a B-cell receptor mimic and essential for B-cell survival. Blood 109:2597-2603, 2007.

[50] Tirelli U, Errante D, Dolcetti R, et al. Hodgkin's disease and human immunodeficiency virus infection: clinicopathologic and virologic features of 114 patients from the Italian Co-operative group on AIDS and Tumors. J Clin Oncol 13:1758-1767, 1995.

[51] Tirelli U, Vaccher E, Serraino D, et al. Comparison of presenting clinical and laboratory findings of patients with persistent generalized lymphadenopathy (PGL) syndrome and malignant lymphoma (ML). Haematologica 72:563-565, 1987.

[52] Andrieu JM, Roithmann S, Tourani JM, et al. Hodgkin's disease during HIV-1 infection: the French registry experience. Ann Oncol 4:635-641, 1993.

[53] Rubio R. Hodgkin's disease associated with human immunodeficiency virus infection. A clinical study of 46 cases. Cancer 73:2400-2407, 1994.

[54] Garcia-Noblejas A, Nieto S, Liberal R, et al. Intracerebral Hodgkin's lymphoma in a patient with human immunodeficiency virus. Haematologica 92:e72-e73, 2007.

[55] Vaccher E, Spina M, Talamini R, et al. Improvement of systemic human immunodeficiency virus-related NHL in the era of HAART. Clin Infect Dis 37:1556-1564, 2003.

[56] Chimienti E, Spina M, Gastaldi R, et al. Clinical characteristics and outcome of 290 patients (pts) with Hodgkin's disease and HIV infection (HD-HIV) in pre and HAART (highly active antiretroviral therapy) era (abstract 168). Ann Oncol 19:iv136, 2008.

[57] Berenguer J, Miralles P, Ribera JM, et al. Characteristics and outcome of AIDS-related Hodgkin's lymphoma before and after the introduction of highly active antiretroviral therapy. J Acquir Immune Defic Syndrome 47:422-428, 2008.

[58] Fazel FB. Hodgkin's Lymphoma at Chris Hani Baragwanath Hospital. A fifteen year review. MMed dissertation. University of the Witwatersrand 2012.

[59] Levine AM, Li P, Cheung T, et al. Chemotherapy consisting of doxorubicin, bleomycin, vinblastine and dacarbazine with granulocyte-colony-stimulating-factor GCSF in HIV infected patients with newly diagnosed Hodgkin's disease: a prospective, multi- 
institutional AIDS Clinical Trials Group Study (ACTG 149). J Acquir Immune Defic Syndr 24:444-450, 2000.

[60] Xicoy B, Ribera JM, Miralles P, et al. Results of treatment with doxorubicin, bleomycin, vinblastine and dacarbazine and highly active antiretroviral therapy in advanced stage human immunodeficiency virus-related Hodgkin's lymphoma. Haematologica 92:191198, 2007.

[61] Hentrich M, Maretta L, Chow JR, et al. Highly active antiretroviral therapy (HAART) improves survival in HIV-associated Hodgkin's disease: results of a multicentre study. Annals of Oncology 17:914-919, 2006.

[62] Spina M, Rossi G, Antinori A, et al. VEBEP regimen and highly active antiretroviral therapy (HAART) in patients (pts) with HD and HIV infection (HD-HIV) (abstract 227). Ann Oncol 19:iv152, 2008.

[63] Hartmann P, Rehwald U, Salzberger B, et al. BEACOPP therapeutic regimen for patients with Hodgkin's disease and HIV infection. Ann Oncol 14:1562-1569, 2003.

[64] Spina M, Gabarre J, Rossi G, et al. Stanford V regimen and concomitant HAART in 59 patients with Hodgkin disease and HIV infection. Blood 100:1984-1988, 2002.

[65] Krishnan A, Molina A, Zaia J, et al. Durable remission with autologous stem cell transplantation for high-risk HIV-associated lymphomas. Blood 105:874-878, 2005.

[66] Spitzer TR, Ambinder RF, Lee JY, et al. Dose-reduced busulfan, cyclophosphamide, and autologous stem cell transplantation for human immunodeficiency virus-associated lymphoma: AIDS Malignancy Consortium Study 020. Biol Blood Marrow Transplant 14:59-66, 2008. 